\title{
«¿DÓNDE VAS A IR TÚ CON SIDA?». INTERSECCIONES ENTRE LA INFECCIÓN POR VIH Y LA VIOLENCIA DEL COMPAÑERO ÍNTIMO
}

\author{
AlberTo MARTíN-PÉREZ RODRÍGUEZ \\ Madrid
}

Recibido/12/09/2011

Aceptado/24/11/2011

\section{Resumen}

Este trabajo consiste en una prospección cualitativa sobre la intersección entre la violencia del compañero íntimo hacia las mujeres (VCI) y la infección por VIH a través de la percepción de siete profesionales de atención sanitaria y de ONG que trabajan con mujeres con VIH y/o con mujeres que sufren VCI en Madrid. Se realizaron entrevistas semiestructuradas y se analizaron los datos a través de procedimientos basados en la teoría fundamentada. Se identificó que la infección por VIH multiplica la violencia psicológica (chantaje, amenaza, desvalorización, etc.) por el estigma asociado a la infección y por algunos efectos secundarios de su tratamiento (lipodistrofia). Los discursos mostraron una visión disociada de los dos problemas de salud pública, lo que indica la necesidad de reconceptualizar la atención y planificación estratégica de ambos.

Palabras clave: VIH, sida, violencia del compañero íntimo (VCI), violencia de género, estigma, mujeres.

\begin{abstract}
This work consists of a qualitative exploration in the intersection of intimate partner violence (IPV) towards women and HIV infection through the perception of seven health care and NGO professionals seeing women with HIV infection and/or women suffering IPV in Madrid. Semi structured interviews were conducted and the data were analyzed using techniques based on the grounded theory. Interviews identified how HIV infection multiplies psychological violence (blackmail, threats, devaluation, etc.) because of HIV social stigmatization and some HIV treatments side effects
\end{abstract}


(lipodystrophy). Professionals' discourses showed a dissasociated vision of both public health problems, which suggests the need to re-conceptualize social and sanitary attention and strategic planning of both IPV and HIV.

Keywords: HIV, AIDS, intimate partner violence (IPV), stigma, women. 


\section{Introducción}

A medida que se combate la violencia del compañero íntimo (en adelante, $\mathrm{VCI}$ ) ésta va adquiriendo visos de mayor sutileza y se requiere profundizar en el conocimiento de sus formas y manifestaciones. En este trabajo se entiende la VCI hacia las mujeres tal como la define la Organización Mundial de la Salud: comportamientos dentro de una relación íntima que causan daños físicos, sexuales o psicológicos, incluyendo agresión física, coacción sexual, abuso psicológico y conductas de control ${ }^{1}$.

Numerosos organismos internacionales y nacionales han señalado la relación que se da entre la violencia contra las mujeres y la infección por VIH. Esta relación puede ser directa, por causas anatómicas y fisiológicas, a través de la violencia sexual o indirecta y por la dificultad de las mujeres que sufren violencia para negociar comportamientos preventivos y acudir a servicios de diagnóstico, prevención y tratamiento, así como por la coerción y manipulación emocional que caracterizan este tipo de relaciones. La percepción de estabilidad que proporciona la relación de pareja, a pesar de la violencia, podría tener más peso que el riesgo asociado a la negociación del preservativo, particularmente en los grupos de mujeres más vulnerables como las usuarias de drogas. No obstante, un reciente estudio no encontró una relación consistente entre la violencia física y sexual y el VIH en mujeres casadas de diez países, lo que indica la necesidad de más investigación para determinar las circunstancias en que dicha relación tiene lugar².

Así mismo, se ha mostrado que las mujeres con infección por VIH pueden estar en riesgo de sufrir violencia tras comunicar su estado serológico a sus compañeros. Para muchas mujeres infectadas, el estigma asociado al VIH implica una caída del ideal patriarcal de «buena mujer» y proveedora de cuidados, resignificándola como «mujer necesitada de cuidados y enferma», sospechosa de haber subvertido mandatos de género, invalidándolas como

1. <http://www.who.int/mediacentre/factsheets/fs239/en/index.html>, consultado el 08-10-2011.

2. HARLING, Guy, et al. «No Association between HIV and intimate partner violence among women in 10 developing countries» PLoS One 5 (2010) e14257. 
mujeres, haciéndolas más violentables y revictimizándolas 3 . Aunque los estudios con muestras concretas - mujeres reclusas, usuarias de drogas intravenosas (UDI), etc.- no indican que las mujeres con VIH sufran más violencia, se ha mostrado que, cuando la sufren, su intensidad y gravedad es más severa.

Algunas autoras han señalado una concurrencia de factores de riesgo comunes para sufrir VCI y para contraer el VIH: tener sexo desprotegido, padecer infecciones de transmisión sexual (ITS), tener múltiples parejas, intercambiar sexo por dinero o drogas, ser usuaria de drogas por vía intravenosa (UDI), tener pareja con VIH o con factores de riesgo para el VIH, revelar el estado serológico, o haber sufrido abusos sexuales en la niñez. Esta enumeración refleja la complejidad de la intersección entre el VIH y la VCI.

Muchos discursos consideran a la mujer más como un vector de la transmisión del VIH que como sujeto del problema y las intervenciones de prevención y cuidados se centran más en otros (hijos e hijas, parejas, clientes...) que en ellas. La mujer queda así invisibilizada como sujeto sexualmente activo y vulnerable ante el VIH. Estos discursos reproducen roles asignados a las mujeres que, para muchas, constituyen imperativos de género que impiden percibir su propia vulnerabilidad y poner en marcha estrategias de prevención. En las relaciones heterosexuales, el aislamiento, la dependencia y la historia previa o actual de violencia de género aumentan el peso de estos condicionantes ${ }^{4}$.

La prevalencia acumulada de VCI en población general mediante encuestas es muy variable por las diferencias metodológicas de los estudios. La encuesta de base poblacional realizada en la Comunidad de Madrid en 2004 con el cuestionario de la encuesta nacional francesa utilizada en el país vecino (ENVEFF), y validado en nuestra población, muestra que el 10,1\% de las mujeres que respondieron a la encuesta había sufrido en el año previo algún tipo de violencia psicológica, física o sexual por parte de su pareja o ex pareja, quedando desagregada la violencia de la siguiente forma: actitudes o conductas de abuso psicológico $(6,8 \%)$, violencia física $(2,4 \%)$, abuso sexual $(0,8 \%)^{5}$.

En la Comunidad de Madrid, el 68,3\% de los casos de sida diagnosticados en mujeres en 2008 se habían debido a la transmisión sexual. El informe del estudio piloto de nuevos diagnósticos de infección por VIH no dispone de

3. GARCIA, M $M^{a}$ Luisa, et al. A pelo gritao. Estudio cualitativo sobre vulnerabilidades, violencias y calidad de vida en mujeres seropositivas. Barcelona, Creación Positiva, 2005. p. 79

4. VelasCo, Sara. Protocolo de prevención de la transmisión heterosexual del VIH con enfoque de género en Atención Primaria. Madrid, Instituto de la Mujer. Ministerio de Trabajo y Asuntos Sociales, 2004. p 19.

5. SANCHEZ, José Luis (coord.). Guía de Apoyo en atención primaria para abordar la violencia de pareja hacia las mujeres. Madrid, Dirección General de Salud Pública y Alimentación. Consejería de Sanidad Comunidad de Madrid, 2008 pp. 10-11. 
información desagregada por sexo para las categorías de transmisión sexual heterosexual y UDI, indicando sólo que las mujeres representan el 19,5\% de los nuevos diagnósticos entre 2007 y $2009^{6}$.

A pesar de su relevancia, la literatura revisada que relaciona el VIH y la VCI evidencia falta de uniformidad en los instrumentos utilizados para medir la VCI. La dimensión psicológica del maltrato suele estar ausente en sus definiciones y utilizan muestras pequeñas y no poblacionales, excepto estudios en África o India y uno más reciente sobre 13.928 mujeres estadounidenses ${ }^{7}$. También son escasos los enfoques cualitativos que caracterizan la violencia de género hacia mujeres con VIH, destacando el de García ${ }^{8}$ que realiza dicha caracterización en un marco de objetivos más amplio.

Combatir la violencia de género es una prioridad de Salud Pública. La investigación y las líneas de trabajo iniciadas sugieren la necesidad de profundizar en las especificidades de la VCI en función de circunstancias personales y sociales como la discapacidad, la inmigración, la edad o el consumo de drogas. La infección por VIH en las mujeres también podría caracterizar de forma diferencial algunas manifestaciones de la VCI. Este trabajo se centra en esta intersección, proponiendo analizar la relación entre VCI y la infección por VIH a través de la percepción de profesionales que trabajan con mujeres en estas situaciones. En concreto se pretende conocer cómo se percibe y se describe la intersección entre la VCI y la infección por VIH, así como identificar oportunidades para un afrontamiento sanitario más efectivo de ambos problemas.

\section{Metodología}

Se realizó una prospección cualitativa empleando como marco epistemológico de género el enfoque integrado de género en salud en sus tres líneas9: la de salud de las mujeres; la de las desigualdades de género en salud y la línea del género como determinante de salud.

El trabajo de campo se realizó a través de entrevistas semiestructuradas, de hora y media de duración, a siete profesionales tomados como informantes

6. <http://www.madrid.org/cs/Satellite?c=PTSA_Multimedia_FA\&cid=1142573182082\& pagename=PortalSalud\%2FPTSA_Multimedia_FA\%2FPTSA_documentoWebeditpro $>$, consultado el 10-08-2010.

7. SAREEN, Jitender, et al. "Is intimate partner violence associated with HIV infection among women in the United States». General Hospital Psychiatry 31 (2009) pp. 274-278.

8. GARCÍA, $\mathrm{M}^{a}$ Luisa, et al. Op.cit.

9. VELASCO, Sara. Sexos, género y salud. Teoría y métodos para la práctica clínica y programas de salud. Madrid, Minerva Ediciones, 2009.

Feminismo/s 18, diciembre 2011, pp. 113-134 
clave por su trabajo con mujeres con infección por VIH y/o con mujeres que sufren violencia, bien en la red de atención sanitaria o en las ONG de Madrid.

Los perfiles profesionales corresponden a una médica de Atención Primaria, un trabajador social de un dispositivo de diagnóstico precoz en un centro de salud, un psicólogo clínico de una unidad hospitalaria de VIH; una médica de un Centro Municipal de Salud para jóvenes, una educadora (con infección por VIH) de un programa de acompañamiento hospitalario entre pares; una psicóloga de una ONG y una trabajadora social de una ONG. Los criterios para la definición de perfiles fueron los de amplitud o máxima diferenciación profesional, y su experiencia en los ámbitos de la VCI, la detección del VIH y la atención psicosocial a mujeres con VIH.

Se eligió a profesionales como informantes en vez de a las mujeres por las dificultades encontradas para acceder a mujeres con infección por VIH que hayan salido de la situación de violencia y por la falta de información de partida sobre algunos aspectos clave que permitieran orientar dichas entrevistas adecuadamente.

Las entrevistas fueron grabadas y transcritas para su análisis posterior. Los campos generales de análisis fueron: la visibilidad de las relaciones entre la VCI y la infección por VIH en la práctica profesional; la percepción social y personal de las mujeres con infección por VIH (imagen, sexualidad, relaciones, etc.) y sus diferencias con los hombres; los hitos relacionados con la enfermedad que pueden desatar episodios violentos; la caracterización de la VCI hacia mujeres con VIH (tipo, manifestación, intensidad...); el afrontamiento de la VCI y autocuidado en las mujeres con infección por VIH; las dificultades para cuidarse/ser cuidada y, por último, las oportunidades y dificultades para el abordaje conjunto de ambos problemas.

El análisis se llevó a cabo mediante procedimientos basados en la teoría fundamentada de Strauss y Corbin ${ }^{10}$, consistente en generar conceptos y categorías para la formulación de hipótesis y teorías a partir del análisis de los datos obtenidos.

Señalamos como limitaciones de este trabajo el pequeño número de profesionales que se entrevistó, y su falta de conocimiento experto simultáneo de VIH y violencia de género, a pesar de su sensibilización ante ambas problemáticas.

10. CORBIN, Juliet; STRAUSS, Anselm. «Grounded theory research: Procedures, canons, and evaluative criteria». Qualitative Sociology; 13 (1990), pp. 3-21. 


\section{Resultados}

En relación con la visibilidad de la intersección estudiada, se apreció que la visión de ambas problemáticas está disociada. Ello se debe a la identificación de la violencia por sus formas más extremas y a la invisibilidad de la violencia sexual dentro de la relación de pareja. Esta última podría, de hecho, ser una forma de VCI reiterada y mantenida en la vida de las mujeres con infección por VIH:

«Lo que pasa es que la gente piensa en la violencia sexual sólo como las violaciones en medio de la calle (...) En nuestras mujeres, al menos de una cierta edad para arriba (...) no se percibe como maltrato pero las relaciones sexuales no consentidas se dan como algo normal en su vida» (Médica de Atención Primaria).

Se mostró que las personas entrevistadas fueron identificando la relación entre VIH y VCI a medida que avanzaba la entrevista. Antes de la misma, se verbalizó la excepcionalidad de la VCI hacia las mujeres con VIH. La entrevista sirvió, así, para visualizar tanto la relación como la cotidianeidad de la violencia hacia estas mujeres:

«Cuando me llamaste para proponerme la entrevista me acordé de casos, de los más gordos, pero la verdad es que luego, pensando, hay un tanto por ciento importante de mujeres maltratadas. Sí. Te das cuenta que ahí ha habido alguna forma de maltrato. Si no un $50 \%$, que yo creo que más, han sufrido maltrato de una forma o de otra» (Psicólogo, Unidad Hospitalaria).

Las y los profesionales explican la vulnerabilidad de las mujeres con infección por VIH ante la VCI por el estigma social asociado a la infección. Este estigma se debe a sus formas de transmisión y a sus implicaciones respecto a los mandatos de género más tradicionales, y constituye una barrera importante para aceptar la enfermedad. Se generan sentimientos como la culpa, la vergüenza y aparece una imagen de sí mismas como «potenciales contaminantes» que refuerza una autopercepción negativa y de baja autoeficacia que ya suele estar presentes en las mujeres que sufren VCI.

«Muchas te dicen todavía que preferirían tener cualquier otra enfermedad, incluso un cáncer, aunque sea más grave, más agresivo para su salud... a nivel psicológico les cuesta más asumir el VIH que otras enfermedades que no son transmisibles, y desde ahí, se complica la asimilación del diagnóstico» (Psicóloga, ONG).

El estigma también puede explicar, al menos en parte, una fase que parece ser común en el proceso de reacción de la mujer infectada ante el diagnóstico y que consiste en negar el problema. Cuando esta fase es más intensa y mantenida en el tiempo, puede tener efectos negativos en la salud e implicar una 
progresión más rápida hacia el sida, ya que la atención sanitaria y social, o la toma de medicación, requieren un cierto nivel de aceptación. Además, tiene efectos en la VCI, puesto que la negación y la consecuente ocultación de la infección aíslan más a la mujer y la hacen más vulnerable al maltrato.

La estigmatización social hacia las mujeres con VIH es causa bien documentada de discriminación social y laboral, así como en el acceso a servicios sociales. En el caso de las mujeres con VIH que sufren VCI, el estigma puede ocasionar problemas de convivencia en recursos de alojamiento compartidos con otras mujeres maltratadas. Así pues, el estigma constituye una importante barrera para salir de la situación de violencia.

«En algunos pisos para mujeres que sufren violencia han puesto pegas por ser seropositivas (...) Por si iban a necesitar vajilla especial» (Trabajadora social, ONG).

«Eso ha pasado en algunos centros (...) porque existe ese miedo a la convivencia en el día a día... no digo que sea generalizado y que otras veces no se vuelquen, pero existe un tanto por ciento de la población que tiene un miedo que (...) es con el que hay que trabajar porque es el que deja a las personas con VIH más desprotegidas» (Psicóloga, ONG).

Un segundo aspecto que aportan las personas entrevistadas para explicar la vulnerabilidad de las mujeres con VIH ante la VCI son los cambios en el aspecto físico debidos a la redistribución de la grasa corporal (lipodistrofia y lipoatrofía facial) y que son efectos secundarios de algunos tratamientos antirretrovirales (TARV). Por su visibilidad y su asociación al VIH, la lipodistrofia (y la anticipación de su aparición) afecta negativamente a la integración laboral de las mujeres, a sus relaciones familiares y sociales o, lo más subrayado en las entrevistas, a su sexualidad y su autoimagen:

«Desnudarse delante de un hombre para algunas mujeres es un trauma. Una mujer (...) que se ve deforme, porque no es que tengas una arruga o dos, es que la lipodistrofia te deforma, pues con eso las mujeres han sufrido un gran shock y de hecho muchas mujeres han decidido no tener más relaciones sexuales» (Educadora de Pares, ONG).

Sin embargo, las mujeres que sufren VCI no suelen estar en posición de renunciar libremente a mantener relaciones sexuales. Y para otras muchas mujeres, la culpa, la inseguridad, los valores negativos incorporados en la identidad a causa de la infección crean un terreno abonado para la violencia y se fuerza la «entrega incondicional» en las relaciones afectivas y sexuales:

«Cuando tienes una relación de pareja, muchas mujeres con VIH asumen cosas con esa pareja que no deberían de asumir, o que no asumirían si no tuvieran $\mathrm{VIH} »$ (Educadora de pares, ONG) 
«Hay casos de mujeres que después de haber sufrido todo lo que sufrieron conocen a alguien, (...) que las trata muy bien y las quiere mucho. De esos casos hay bastantes. Y además que les cuesta que las puedan querer sin más» (Psicólogo, Unidad Hospitalaria).

Esta última frase muestra cómo muchas mujeres interiorizan la idea de que la infección por VIH les resta valor y las hace menos deseables y dignas de amor; ésta es una línea argumental que emplea el maltratador hacia la mujer con VIH.

Tanto por la aceptación de una enfermedad estigmatizada como por la dificultad percibida para establecer relaciones afectivas y de pareja futuras, se manifestó que las mujeres con VIH necesitan y demandan más apoyo psicosocial que los hombres, a pesar de la menor prevalencia de la infección en mujeres. Este tipo de apoyo psicosocial se señala, de hecho, como una estrategia de cuidados de gran importancia.

«A nivel de la unidad por completo igual hay más hombres que mujeres, pero a nivel de atención psicológica yo creo que la cosa está al 50\%» (Psicólogo, Unidad Hospitalaria)

«El número de sesiones que necesitan para asumir su enfermedad en un reciente diagnóstico, (...) a las mujeres les cuesta más, está claro. Y les cuesta más cuando no tienen pareja o cuando vienen de una ruptura (...) Hace poco una chica (...) que está rompiendo con la pareja me decía «creo que no estaría tan mal por la separación en este momento si no tuviera VIH». Encarar la búsqueda de una nueva relación se les hace más duro». (Psicóloga, ONG)

En relación con los autocuidados se indicó la idea de que la mujer con VIH se cuida para cuidar, en particular si existen otros casos de infección en la unidad familiar. Se incidió en que las mujeres con infección por VIH que sufren VCI son las que tienen más dificultades para cuidarse, en cualquier forma, mientras están en la relación de maltrato. Muchos autocuidados (la toma de la medicación, acudir a grupos de ayuda mutua, etc.) implican una cierta aceptación de la infección por VIH y una cierta visibilidad de la condición de seropositiva frente a terceros que pueden quedar comprometidas en situaciones de mayor aislamiento a causa de la violencia. El abordaje de la VCI y de sus efectos que fue planteado implicaría las mismas estrategias que se seguirían con cualquier mujer que sufra VCI, teniendo en cuenta lo específico del VIH:

«Trabajar con la visibilidad con las personas íntimas que la vayan a poder proteger, trabajar con la autoestima, crear lazos con otras mujeres con infección por VIH, trabajar la información, habilidades sociales (...) Es decir, en parte, además de lo relacionado con el VIH, el trabajo que se hace con una persona en situación de violencia» (Psicóloga, ONG).

Feminismo/s 18, diciembre 2011, pp. 113-134 
Respecto a cómo se manifiesta la VCI hacia las mujeres con VIH, las agresiones físicas parecen relacionarse en mayor medida con los casos en que la mujer, la pareja o ambos son consumidores de drogas mientras que en otros casos, aunque también pueda coexistir violencia física y sexual, es más frecuente la psicológica ya que la infección es utilizada como instrumento para ejercerla, como se muestra a continuación.

- Amenaza, chantaje, control y aislamiento: revelar el estado serológico a la pareja se configura como un momento crítico para la aparición del maltrato. Las dificultades señaladas para asimilar el diagnóstico a causa del estigma y el deseo de la mujer de ocultar su estado serológico permiten al maltratador chantajear a la mujer a través de amenazas de revelar a terceros, en distintos entornos, su condición de seropositiva, así como la de sus hijos o familiares en caso de que también tuvieran VIH. Además del efecto que esto tiene en el control de las relaciones sociales de la mujer y en su aislamiento, el coste social para ella, si la amenaza se cumple, puede ser altísimo:

«Destruir tu entorno social, donde tú no quieres que se sepa; en el restaurante donde comes cada día (...) «ten cuidado con ésta que tiene sida, no vaya a ser que...» (...) Y eso te mata socialmente. No te mata físicamente, pero te mata socialmente» (Educadora de Pares, ONG).

El chantaje será más viable cuanto más difícil sea para la mujer hacer visible hacia su entorno su estado serológico frente al VIH. Por ello, estas mujeres seguirían siendo vulnerables ante la violencia ejercida por sucesivas parejas:

«También es verdad (...) que estos maridos ya han fallecido a veces pero ellas siguen haciendo una vida en la que hubo una barrera y les cuesta mucho (...) relacionarse con su familia de origen, que hablen de lo que pasó, que puedan volver a abrirse» (Psicólogo Unidad Hospitalaria).

Ésta parece ser una forma de maltrato hacia las mujeres con infección por VIH comúnmente identificada y que la expansión de las nuevas tecnologías de la información y las redes sociales en Internet parecen haber potenciado. Su visibilidad en los discursos de las y los profesionales se debe a que probablemente tiene lugar cuando la mujer contacta con el sistema sanitario para un seguimiento clínico, farmacológico o para recibir apoyo psicosocial, a través del cual puede tomar conciencia de la relación de maltrato, y se encuentra en fases de preparación y/o acción para salir de la misma: 
«Cuando ella decide romper el vínculo e irse de casa estaba trabajando, cuidando a una persona en una casa, y él va y llama a la casa para decir que ella tiene VIH; (...) tener una información sobre ti y utilizarla como quiera si no estás conmigo» (Psicólogo Unidad Hospitalaria)

«El que abusa o maltrata utiliza todo lo utilizable, como otros aspectos de tu vida, o tal, el temor ¿no?, que tienen muchas mujeres de que se cuente esto o lo otro» (Médica de Atención Primaria).

En ocasiones, cuando la mujer está emprendiendo pasos para salir de una situación de violencia necesita ocultar al maltratador su diagnóstico, porque se percibe que la infección será un motivo más para el maltrato:

«Hay un caso en que ella pide un informe para pedir una orden de alejamiento y en ese informe (...) no tengo que poner nada de que ella tiene VIH, porque no quiere que él sepa que tiene VIH. Sabe que él se lo ha transmitido pero no quiere que él lo sepa por la posibilidad de que haya malos tratos» (Psicólogo, Unidad Hospitalaria).

Paradójicamente, en algunos casos, cuando se revela a la familia el estado serológico de la mujer, aunque ella no lo haya decidido así, el efecto, al desaparecer el peso de la amenaza, podría ser el contrario al perseguido por el maltratador y proporcionar una válvula que permita salir del aislamiento:

«Pues mira, ¿la verdad? se sintió relajada. Es curioso. «Muy bien, pues ahora que me siento obligada, yo por mi no lo habría dicho, pero ahora lo cuento, y tengo la oportunidad de hacerlo». Ella decía «es que estoy como liberada, porque lo he podido hablar con ellos, él casi me ha hecho un favor». Porque antes, lo que estaba haciendo él era, metiéndole miedo de contarlo, retenerla para que no le dejara. Recortando su relación con los familiares» (Trabajadora Social, ONG)

Este comentario muestra la importancia de favorecer la aceptación y dotar de estrategias a las mujeres para la revelación voluntaria y segura del diagnóstico ya que dar a conocer su situación puede ser una estrategia de protección frente al aislamiento.

- La incapacitación para la acción: cuando el maltrato está instaurado en la relación antes de la infección por VIH de la mujer o de su revelación a la pareja, la infección puede utilizarse para mantener a la mujer en dicha situación reforzando la idea de que, por sus implicaciones sociales, el VIH la incapacita para entrar en fases de preparación y acción que son previas a la salida de la situación de violencia, como indican Prochaska y Di Clemente 
en su modelo espiral de las etapas de cambio ${ }^{11}$. La diferencia con otras enfermedades crónicas es el estigma asociado al VIH que refuerza la sujeción de la mujer a la situación de violencia.

«Si muchas veces las preguntas serían «si le dejo, ¿dónde voy?, ¿cómo voy a mantener a mis hijos?, ¿dónde voy a trabajar, si no soy nadie»... Pues si a eso le añades el VIH aún condiciona más ¿no? Además es muy repetitivo por parte de los hombres en algunos casos de violencia de género: «¿dónde vas a ir tú, con sida?» (Educadora de Pares, ONG).

«Lo que ha hecho el diagnóstico es acrecentar esa violencia (...); saca al hombre una justificación más para ser violento y a ella una justificación para dejarse ser violentada (...) sabes todos los condicionantes sociales que conlleva tener VIH y ser mujer y que piensas que nadie te va a querer, que bastante es que él te quiera» (Educadora de Pares, ONG).

- La desvalorización: otro momento crítico para la aparición de VCI hacia estas mujeres ocurre cuando se manifiestan los efectos de la enfermedad o los efectos secundarios de algunos TARV, como la lipodistrofia, que aparte de su gravedad clínica y social se convierte en argumento para el maltrato a través del insulto y la descalificación que conducen a la desvalorización de la mujer.

«El insulto relacionado con el VIH: «sidosa, me das asco». La agresión verbal utilizando el VIH de forma directa " $i$ a ti quien te va a querer con lo que tienes? Ahora sólo puedes estar conmigo», utilizando el VIH como elemento fundamental de la agresión» (Psicóloga, ONG).

«Cuando se toma la medicación puede ser un momento de crisis en la evolución de la enfermedad. Y en el caso de personas que han sufrido cierto maltrato psicológico se me ocurre que puede tener que ver con la aparición de efectos secundarios, físicos, como la lipodistrofia y viene el «es que fíjate como estás, vaya piernas que tienes, ¿dónde vas con esa falda?», "ies que no te ves el cuerpo que tienes, dónde vas con eso?» (Psicóloga, ONG).

- Separación de hijos e hijas: se señaló cómo algunos maltratadores limitan el contacto entre la mujer con VIH y sus hijos, argumentando el riesgo de infección, por desconocimiento sobre las vías reales de transmisión o con la intención expresa de causar un daño a la mujer.

«Por ejemplo que el marido no las deje secarse con las toallas con las que se secan los hijos y cosas de esas, ¿no? (...) tienen una enfermedad infecto-contagiosa, (...) la información que reciben no les tranquiliza

11. Prochaska, James, et al. «Cómo cambia la gente. Aplicaciones en los comportamientos adictivos». RET. Revista de Toxicomanías 1 (1994), pp. 1-12. 
respecto a que puedan transmitírselo a sus hijos (...) y si a eso le añades que la pareja, (...) constantemente se lo está diciendo, pues esa creencia aumenta» (Educadora de pares, ONG)

En relación con el segundo objetivo que se perseguía de identificar oportunidades para afrontar de forma conjunta la VCI hacia las mujeres y la infección por VIH, el discurso de las personas entrevistadas sugirió que dicha integración podría realizarse a través de diversas estrategias en el contexto del diagnóstico de la infección por VIH y de la atención sanitaria a las personas con $\mathrm{VIH}^{12}$.

Por otra parte, se señaló la importancia de sensibilizar e informar al personal de todas las redes de recursos de atención a las mujeres que sufren violencia sobre la imposibilidad de transmisión del VIH en las condiciones comunes de la vida cotidiana, y lo injustificado de discriminar a las mujeres con VIH en estos recursos y servicios.

Así mismo, se señaló la conveniencia de sensibilizar y formar a las y los profesionales que trabajan en la detección y el abordaje de la violencia de género sobre las necesidades clínicas y psicosociales de las mujeres con VIH y sobre la especificidad de la VCI a la que son sometidas. También se señaló la importancia de visibilizar la vulnerabilidad frente al VIH de las mujeres mayores que sufren VCI:

«Esta señora que es la del marido que toda la vida, que es un hijo de tal, que ha sido un mal hombre por decirlo así, probablemente, vamos, con seguridad, ha tenido otras relaciones y si no dan signos de tener VIH, ni se lo hacemos a él, ni a ella. Lo asociamos a vida sexual activa» (Médica de Atención Primaria)

\section{Discusión}

A diferencia del estudio de García et al ${ }^{13}$ que infería de los discursos de las mujeres con infección por VIH entrevistadas la ausencia de una reflexión crítica sobre la violencia por parte de las y los profesionales que las atienden, en nuestros resultados se detecta esta asociación entre la VCI hacia las mujeres y el VIH. No obstante, la asociación no es espontánea sino que se va identificando más claramente a medida que avanza la entrevista. La percepción de excepcionalidad de la intersección previa a la entrevista se explica por la

12. MARTín-PÉREZ, Alberto, et al. «Detección de la violencia de pareja en las intervenciones diagnósticas y de atención sanitaria a mujeres con VIH en Madrid». Programa del XIV Congreso Nacional sobre el Sida. Infección por VIH, 30 años después Jun 15-17, Zaragoza (2011), P5.29.

13. GARCIA, Ma Luisa, et al. Op.cit., p. 79.

Feminismo/s 18, diciembre 2011, pp. 113-134 
identificación de la violencia por sus manifestaciones más extremas, quedando ocultos la violencia psicológica y el sexo no consentido en pareja, como en la mayoría de la literatura consultada. Sin embargo, la dimensión psicológica del maltrato es posteriormente la más señalada en los discursos. Todo ello apunta la necesidad de potenciar el afrontamiento conjunto de ambos problemas, hecho que puede lograrse desde la formación de profesionales y/o la adecuación de los instrumentos de registro de casos en los diferentes ámbitos de atención a estas mujeres.

No es posible concluir sobre las características sociodemográficas de las mujeres más afectadas por esta intersección a partir de los resultados de este trabajo, pero el eco común en los discursos es el eje de la marginalidad y la exclusión: las y los profesionales entrevistados hacen referencia a mujeres inmigrantes y jóvenes, UDI, mujeres españolas parejas de UDI o de inmigrantes. Se señala sin embargo, la invisibilidad de un grado más alto en la exclusión, pues llama la atención en los discursos la ausencia de referencia a las mujeres que ejercen prostitución y a las transexuales, muy vulnerables tanto a la infección como a la violencia ${ }^{14}{ }^{1516}$.

La aceptación de la infección, que influirá en los cuidados, en el inicio y adherencia a los TARV y, a la larga, en el pronóstico terapéutico, parece relacionarse más con los significados sociales de esta enfermedad que con los puramente clínicos. Los avances en los TARV y en la atención han hecho de la infección por VIH en el mundo occidental una enfermedad crónica y compatible con una esperanza de vida casi normal, mejorando la calidad de vida de las y los pacientes, y dificultando la transmisión del virus. Sin embargo, la percepción social del VIH ha sufrido pocos cambios significativos. Su estigmatización se explica por sus vías de transmisión y sus implicaciones respecto a los mandatos de género más tradicionales y a los atributos y exigencias de género más contemporáneos (belleza, disposición sexual, etc.) definitorios de la feminidad. Tener VIH supone un obstáculo más para que las mujeres que no han realizado un planteamiento crítico de este ideal inalcanzable de feminidad sientan que se acercan al mismo. Además, sigue siendo motivo

14. EL-BASSEL, Nabila, et al. «Correlates of partner violence among female street-based sex workers: substance abuse, history of childhood abuse and HIV risks». AIDS Patient Care and STDs 15 (2001) pp. 41-51.

15. ClEMENTS-NOLLE, Kristen, et al. «HIV prevalence, risk behaviours, health care use and mental health status of transgender persons. Implications for Public Health interventions». Am J Pub Health 9 (2001), pp. 915-921.

16. XAVIER, Jessica, et al. «An overview of US trans health priorities. A report by the Eliminating Disparities Working Group». National Coalition for LGTB Health; Washington (2004). 
de discriminación en distintos ámbitos. Esto explicaría la superior demanda identificada de apoyo psicosocial en mujeres y el mayor índice de mujeres negadoras. La negación aísla a la mujer de las redes sanitarias y sociales, haciéndola más vulnerable ante la VCI. En este sentido, la literatura relaciona la VCI hacia las mujeres con VIH con una carga viral elevada, menor recuento linfocitario y peor adherencia a los tratamientos. Esto a su vez, aumentaría el riesgo de discapacidad, sumando otro factor facilitador de la violencia.

El significado subjetivo de perder la belleza exigida a la mujer, en este caso por la enfermedad y/o por la lipoatrofia facial y lipodistrofia, pueden mantener a las mujeres en posiciones pasivas y sin oportunidad de hacer frente a la desvalorización. Unido a las dificultades señaladas para aceptar la enfermedad, al sentimiento de culpa y a la crisis por no poder responder a las exigencias de feminidad, puede causar mayor inseguridad y sumisión al varón, cuya imagen se sublima como alguien en quien refugiarse.

Parece, pues, recomendable abordar la asertividad y trabajar con la imagen corporal para afrontar las exigencias sociales de belleza y seducción con las mujeres que sufren lipodistrofia. Puede mejorar su calidad de vida, ya que la anticipación de la lipodistrofia podría disuadir de la toma de medicación y dificultar la adherencia. También puede facilitar el afrontamiento de la VCI centrada en la humillación derivada de estos efectos secundarios. Sin olvidar, en todo caso, su efecto real en la discriminación en distintos ámbitos y la necesidad de poner el acento en la propia situación de maltrato y no sólo en la autoestima de la mujer. La asociación entre la lipodistrofia y la VCI constituye un argumento más para la investigación en TARV con menos efectos secundarios, y para garantizar la igualdad en el acceso a las cirugías reparadoras, que está actualmente en uso tutelado en nuestro país, con el fin de valorar su inclusión en el catálogo de prestaciones del Sistema Nacional de Salud.

Valorar ventajas e inconvenientes de revelar el estado serológico frente al VIH en el entorno cercano para romper el aislamiento, pero sin comprometer la seguridad, podría ser una línea de trabajo con mujeres en fases de acción para salir de la situación de maltrato. Los inconvenientes pueden, en algunos casos, sobredimensionarse y mantener operativo el chantaje del maltratador.

Los resultados coinciden con la literatura en señalar la infección por VIH como un multiplicador de la violencia de género. La enfermedad o la discapacidad, en general, hacen a la mujer más vulnerable ante la VCI y la misma infección por VIH proporciona al maltratador argumentos específicos (chantaje, aislamiento, incapacitación para la acción, insulto e impedimento del contacto con hijos e hijas) para producir maltrato psicológico aunque éste estuviera presente antes de la infección. Esto subraya la importancia de entender la 
bidireccionalidad de la relación entre VIH y VCI, ya que en muchos casos será dicha violencia la que explique la infección de la mujer. García et al añade el rechazo $0^{17}$ de la pareja a la caracterización de la violencia cuya anticipación podría relacionarse también con la renuncia a las relaciones sexuales y afectivas identificada en nuestros resultados.

Para afrontar la intersección de la VCI y el VIH, algunas experiencias en otros países muestran la posibilidad de integrar la detección de VCI en servicios de diagnóstico de infección por VIH durante las sesiones de pre y post counseling de una cierta duración ${ }^{18}{ }^{19}$. Además, las unidades hospitalarias, por su contacto continuado con las y los pacientes con infección por VIH se configuran como un espacio en el que, de disponer de unidades de apoyo interdisciplinar, se podría abordar la VCI además de otras intervenciones de prevención y de mejora de la calidad de vida. Hay evidencias de que las mujeres con infección por VIH que sufren VCI muestran más episodios de hospitalización y peor pronóstico y tienen peores índices de salud mental, lo que aconseja implicar a la atención especializada. Además, incluir servicios relacionados con la violencia de género en el contexto de la atención a las personas con VIH muestra que su abordaje no es un tabú y que las y los profesionales de la sanidad están en posición de ayudar a las mujeres a salir de la situación de maltrato.

La discriminación hacia las mujeres con infección por VIH en los recursos y servicios sociales para mujeres que sufren VCI depende no sólo de las y los profesionales que la aplican sino de las instituciones que la sustentan. La décima Clasificación Internacional de Enfermedades (CIE) de la Organización Mundial de la Salud (OMS) codifica la infección por VIH en «diseases generally recognised as communicable or transmissible $»^{20}$. La traducción de la CIE realizada por la Organización Panamericana de la Salud (OPS) traduce, en nuestra opinión inadecuadamente, por «infecto-contagioso» ${ }^{21}$. De forma contradictoria, la propia OPS recomienda utilizar «transmisible» en vez de

17. GARCíA, Ma Luisa, et al. Op.cit., p. 64.

18. New York State Department of Health. «Guidelines for Integrating Domestic Violence Screening into HIV Counseling, Testing, Referral \& Partner Notification». Disponible en http://www.health.state.ny.us/nysdoh/rfa/hiv/guide.htm, consultado el 07-07-2010.

19. KLEIN, Susan, et al. «Screening persons newly diagnosed with HIV/AIDS for risk of intimate partner violence: early progress in changing practice». J Public Health Management Practice 14 (2008), pp. 420-428.

20. World Health Organization. Internacional Statistical Classification of Diseases and Related Health Problems. 10 Revision. Version for 2007. Disponible en http://apps.who.int/ classifications/apps/icd/icd10online/, consultado el 15-08-2010.

21. Pan American Health Organization. Clasificación Estadística Internacional de Enfermedades y Problemas Relacionados con la Salud (CIE-10). Caracas, 2008. 
«contagioso» para referirse al VIH por la carga moral de este último término y por sugerir que la transmisión es fácil ${ }^{22}$. Lingüística y médicamente el término «contagioso» puede ser correcto, pero se asocia con algo fuera del control humano, provocando miedos y situaciones de evitación injustificados ya que la evidencia científica muestra que la transmisión del VIH no se produce en actos de la vida cotidiana. Más allá de garantizar la no discriminación en estos recursos, se debería favorecer su función de apoyo a la adherencia a los TARV, así como la prevención de la infección por VIH dirigida a todas las mujeres usuarias de estos recursos, como muestran algunas experiencias identificadas en la literatura.

Por último, parece adecuado incorporar en los programas de sensibilización, formación y abordaje de la violencia de género la reflexión sobre las intersecciones de la VCI con la infección por VIH, en ambos sentidos. Para ello es necesario sumar voces como indican estos resultados y otras declaraciones y artículos revisados.

\section{Conclusiones}

Treinta años después de la aparición de los primeros diagnósticos de infección por VIH, la falta de visibilidad de su intersección con la VCI indica la necesidad de reconceptualizar la atención y la planificación estratégica de ambos problemas de Salud Pública, de establecer alianzas y de adaptar los sistemas de información y vigilancia epidemiológica actuales, como la Encuesta Hospitalaria de pacientes con VIH/sida, las encuestas regionales de salud o las encuestas de violencia, para poder dimensionar el problema.

La infección por VIH puede incrementar la vulnerabilidad de las mujeres ante la VCI y se puede utilizar para articular formas de maltrato psicológico sostenidas por el estigma asociado a la infección o por los efectos secundarios de su tratamiento.

Para combatir este estigma es necesario sensibilizar sobre la utilización del término «infecto-contagioso» para referirse a la infección por VIH en informes médicos y proponer alternativas, así como garantizar que su catalogación en el CIE no produzca discriminación en ningún tipo de servicio o recurso social ya que no existen evidencias que la justifiquen. El efecto de la lipodistrofia en la calidad de vida y en la VCI indica la necesidad de trabajar con las mujeres aspectos relacionados con la imagen corporal y la autoestima

22. Pan American Health Organization. «Terminología relacionada con el VIH: actualización 2006 de la OPS». 2006. Disponible en http://www.cisas.org.ni/files/vih.pdf, consultado el 15-08-2010. 
en los niveles de atención donde sea posible, así como de apoyar el trabajo de las ONG para la ayuda mutua y empoderamiento de las mujeres con VIH.

Aumentar la visibilidad de esta intersección y realizar un abordaje integrado requiere sensibilizar y formar a las y los profesionales que intervienen en los programas de diagnóstico de VIH y atención a las mujeres con VIH para la detección y abordaje de la $\mathrm{VCI}$, así como adecuar los protocolos y guías de actuación sobre la prueba rápida del VIH. Igualmente sería deseable la adecuación de documentos técnicos y formativos sobre violencia de género para visibilizar tanto la vulnerabilidad específica de las mujeres con infección por VIH como el riesgo de infección que implica la VCI, insistiendo particularmente en la vulnerabilidad, más oculta, de las mujeres mayores.

Es necesario profundizar en el conocimiento de algunos elementos básicos de esta intersección desde la percepción de las propias mujeres con VIH que sufren o han sufrido $\mathrm{VCI}$, como pueden ser el efecto de la devastación causada por la violencia en el pronóstico de la infección, la adherencia al TARV, los cuidados y rutinas en salud, etc., teniendo en cuenta la causalidad y efectos acumulativos de la concurrencia de VCI, otras manifestaciones de la violencia de género, el VIH, el uso de drogas y la salud mental.

\section{Referencias bibliográficas}

AguirRezABAL, Arrate; Fuster, Ma José; VAlEnCia, José. Integración laboral de las personas con VIH. Estudio sobre la identificación de las necesidades laborales y la actitud empresarial. Madrid, Fundación para la investigación y la prevención del Sida en España, 2009.

American Foundation for AIDS Research. Gender-Based Violence and HIV Among Women: Assessing the Evidence. Issue Brief No. 3. Washington, AmFAR, 2005.

BoILY, Marie Claude; BUVÉ, Anne; BAGGALEY, Rebecca. «HIV transmission in serodiscordant heterosexual couples». BMJ 340 (2010), c2449.

Clements-Nolle, Kristen; MARX, Rani; GuZMan, Robert; KatZ, Mitchell. «HIV prevalence, risk behaviours, health care use and mental health status of transgender persons. Implications for Public Health interventions». Am J Pub Health 9 (2001), pp. 915-921.

Cohen, Mardge; Deamant, Catherine; Barkan, Susan; Richardson, Jean; YOUNG, Mary; HOLMAN; Susan, et al. «Domestic violence and childhood sexual abuse in HIV infected women and women at risk for HIV». Am J Pub Health 90 (2000), pp. 560-565.

CORBIN, Juliet, STRAUSS, Anselm. "Grounded theory research: Procedures, canons, and evaluative criteria». Qualitative Sociology; 13(1) (1990), pp. 3-21. 
DaVILA, Yolanda; Bonilla, Elsa; GonZÁleZ-RamíREZ, Dolores; Grinsdale, Susan; Villarruel, Antonia. «Pilot testing HIV and intimate partner violence prevention modules among spanish-speaking latinas». JANAC 19 (2008), pp. 219-224.

El-BASSEL, Nabila; WitTe, Susan; WADA Takeshi; GILBERT, Louisa; WALlACE, Joyce. «Correlates of partner violence among female street-based sex workers: substance abuse, history of childhood abuse and HIV risks». AIDS Patient Care and STDs 15 (2001) pp. 41-51.

El-BAsSel, Nabila; Gilbert, Louisa; WU, Elwin; GO, Hyun; Hill, Jennifer, Hill. «Relationship between drug abuse and intimate partner violence: a longitudinal study among women receiving methadone». Am J Pub Health 95 (2005), pp. 465-470.

El-BASSEL, Nabila; Gilbert, Louisa; Wu, Elwin; Chang, Mingway; GoMes, Carla; VINOCOUR, Danielle, et al. «Intimate partner violence prevalence and HIV risks among women receiving care in emergency departments: implications for IPV and HIV screening» Emerg Med J 24 (2007), pp. 255-59.

García, Ma Luisa; Maragall, Margarita; Pineda, Montserrat; SolÉ, Nuria; VÁzQUEZ, Ma José. A pelo gritao. Estudio cualitativo sobre vulnerabilidades, violencias y calidad de vida en mujeres seropositivas. Barcelona, Creación Positiva, 2005.

GIElen, Andrea C; O'CAMPO, Patricia; FAdEN, Ruth; EKE, Agatha. «Women's disclosure of HIV status: experiences of mistreatment and violence in an urban setting». Women \& Health 25 (1997), pp. 19-31.

Gielen, Andrea C; Fogarty, Linda; O Campo, Patricia; Anderson, Jean; Keller, Jean; FADEN, Ruth. «Women living with HIV: disclosure, violence and social support». J Urban Health 77 (2000), pp. 480-491.

GIELEN, Andrea C. "Sexual violence and abuse in intimate relationships», en AmFAR: Women, Sexual Violence and HIV. New York, The American Foundation for AIDS Research, 2005, pp 9-11.

Gielen, Andrea C; MCDOnnell, Karen; O'CAMPo Patricia; Burke, Jessica. «Suicide risk and mental health indicators: do they differ by abuse and HIV status?» Women's Health Issues 15 (2005), pp. 89-95.

Gielen, Andrea C; Ghandour, Reem; Burke, Jessica; Mahoney, Patricia; MCDONNELL, Karen; O'CAMPO, Patricia. «HIV/AIDS and intimate partner violence: Intersecting women's health issues in the United States». Trauma, Violence, \& Abuse 8 (2007), pp. 178-198.

Global Coalition on Women and AIDS. Stop violence against women. Fight AIDS. What's real. Issue 2. Ginebra, 2005. Disponible en: http://data.unaids.org/Publications/IRC-pub07/jc1184-stopviolence_en.pdf, consultado el 15-09-2010.

Gruskin, Leslie; GAnge, Stephen; Celentano, David; Schuman, Paula; Moore, Janet; ZIERLER, Sally, et al. «Incidence of Violence against HIV-infected and 
uninfected women: findings from the HIV Epidemiology Research Study».J Urban Health 79 (2002), pp. 512-524.

Fuster, Ma José; Molero, Fernando; Gil, Lorena; Vitoria, Amaia, AguirrezABAL; Arrate; Kindelan, Antonio. Creencias y actitudes de la población española hacia las personas con VIH. Madrid, Fundación para la Investigación y la Prevención del Sida en España, 2010.

Harling, Guy; Msisha, Wezi; Subramanian S.V. «No Association between HIV and intimate partner violence among women in 10 developing countries». PLoS One 5 (2010), el4257.

Instituto de Derechos Humanos Bartolomé de las Casas. Universidad Carlos III, Madrid. Discriminación y VIH/SIDA 2005. Estudio FIPSE sobre discriminación arbitraria de las personas que viven con VIH o SIDA. Madrid, Fundación para la Investigación y la Prevención del Sida en España, 2005.

Jones, Allison S; Lillie-Blanton, Marsha; Stone, Valerie; Ip, Edward; ZHAng, Qiang; WILSON, Tracey. «Multi-dimensional risk factor patterns associated with non-use of highly active antiretroviral therapy among human immunodeficiency virus-infected women». Women's Health Issues 20 (2010), pp. 335-342.

Klein, Susan; Tesoriero, James; Leung, Shu-Yin J; Heavner, Karin; BirkHeAd Guthrie. «Screening persons newly diagnosed with HIV/AIDS for risk of intimate partner violence: early progress in changing practice».J Public Health Management Practice 14 (2008), pp. 420-428.

LAUGHON, Kathryn. «Abused African American women's processes of staying healthy». Western Journal of Nursing Research 29 (2007), pp. 365-384.

Liebschutz, Jane; Feinman, Gail; Sullivan, Lisa; Stein, Michael; Samet, Jeffrey. «Physical and sexual abuse in women infected with the human immunodeficiency virus. Increased illness and health care utilization». Arch Intern Med 160 (2000), pp. 1659.1664.

Martín-PÉREZ, Alberto; Lasheras, Luisa; Pires, Marisa. «Detección de la violencia de pareja en las intervenciones diagnósticas y de atención sanitaria a mujeres con VIH en Madrid». Programa del XIV Congreso Nacional sobre el Sida. Infección por VIH, 30 años después Jun 15-17, Zaragoza (2011), P5.29.

Medley, Amy; García-Moreno, Claudia; McGill, Scott; Maman, Susan. «Rates, barriers and outcomes of HIV serostatus disclosure among women in developing countries: Implications for prevention of mother-to-child transmission programmes». Bull World Health Organ 82 (2004), pp. 299-307.

New York State Department of Health. «Guidelines for Integrating Domestic Violence Screening into HIV Counseling, Testing, Referral \& Partner Notification». Disponible en http://www.health.state.ny.us/nysdoh/rfa/hiv/guide.htm, consultado el 07-07-2010 
Pan American Health Organization. «Terminología relacionada con el VIH: actualización 2006 de la OPS». 2006. Disponible en http://www.cisas.org.ni/files/ vih.pdf, consultado el 15-08-2010.

Pan American Health Organization. Clasificación Estadística Internacional de Enfermedades y Problemas Relacionados con la Salud (CIE-10). Caracas, 2008.

Prochaska, James; Di Clemente, Carlo; Norcross, John. «Cómo cambia la gente. Aplicaciones en los comportamientos adictivos». Revista de Toxicomanías 1 (1994) pp. 1-12.

Romero, Jorge del; Castilla, Jesús; Hernando, Victoria; Rodríguez, Carmen; GARCÍA, Soledad. «Combined antiretroviral treatment and heterosexual transmisión of HIV-1: cross sectional and prospective cohort study». BMJ 340 (2010), c2205.

Rose, Rachel; House, Amy, StePleman, Lara. «Intimate Partner Violence and its effects on the Health of African American HIV Positive Women». Psychological Trauma: Theory, Research, Practice and Policy 2 (2010), pp. 311-317.

Rountree, Michele; Pomeroy, Elizabeth; Marsiglia, Flavio. «Domestic violence shelters as prevention agents for HIV/AIDS?» Health \& Social Work 33 (2008), pp. 221-28.

Ruíz-PÉRez, Isabel; Plazaola-CAStaÑo, Juncal, Vives-CASES, Carmen. «Methodological issues in the study of violence against women».J Epidemiol Community Health 61 (2007), pp. 26-31.

SÁnCHEZ, José Luis; Pires, Marisa; EsCortell, Esperanza; DOMíngueZ, Julia; LASHERAS, María Luisa; LóPEZ, Sonia. Guía de Apoyo en atención primaria para abordar la violencia de pareja hacia las mujeres. Madrid, Dirección General de Salud Pública y Alimentación. Consejería de Sanidad Comunidad de Madrid, 2008.

SAREEN, Jitender; PAGURA, Jina; GRANT, Bridget. «Is intimate partner violence associated with HIV infection among women in the United States?». General Hospital Psychiatry 31 (2009), pp. 274-278.

TufTS, Kimberly; Clements, Paul T; Wessell, Judy. «When intimate partner violence against women and HIV collide: challenges for healthcare assessment and intervention». J Forensic Nurs 6 (2010), pp. 66-73.

Velasco, Sara. Protocolo de prevención de la transmisión heterosexual del VIH con enfoque de género en Atención Primaria. Madrid, Instituto de la Mujer. Ministerio de Trabajo y Asuntos Sociales, 2004.

Velasco, Sara. Sexos, género y salud. Teoría y métodos para la práctica clínica y programas de salud. Madrid, Minerva Ediciones, 2009.

World Health Organization. Gender Dimensions of HIV Status Disclosure to Sexual Partners: Rates, Barriers, Outcomes. A review paper. Geneva, WHO, 2003. 
World Health Organization. Internacional Statistical Classification of Diseases and Related Health Problems. 10 $0^{\text {th }}$ Revision. Version for 2007. Disponible en http:// apps.who.int/classifications/apps/icd/icd10online/, consultado el 15-08-2010. Xavier, Jessica; Hitchcock, Donald; Hollinshead, Susan, Keisling, Mara; Lewis, Yosenio; LOMBARDI, Emilia, et al. «An overview of US trans health priorities. A report by the Eliminating Disparities Working Group». National Coalition for LGTB Health; Washington (2004). Disponible en: http://www.transequality. org/PDFs/HealthPriorities.pdf

Zierler, Sally; Cunnigham, William; Andersen, Ron; Shapiro, Martin; BozzetTER, Sam; NAKAZONO, Terry, et al. «Violence victimization after HIV infection in a US probability sample of adult patients in Primary Care». Am J Pub Health 90 (2000), pp. 208-215. 